\title{
Assessing the impact of next-generation rapid diagnostic tests on Plasmodium falciparum malaria elimination strategies
}

\author{
Hannah C. Slater*1, Amanda Ross*2,3, André Lin Ouédraogo ${ }^{4,5}$, Lisa J. White ${ }^{6,7}$, Chea Nguon ${ }^{8}$ Patrick G.T. Walker ${ }^{1}$, \\ Pengby Ngor ${ }^{6,8}$, Ricardo Aguas ${ }^{6}$, Sheetal P. Silal ${ }^{9}$, Arjen M. Dondorp ${ }^{6,7}$, Paul La Barre ${ }^{10}$, Robert Burton ${ }^{10}$, \\ Robert W. Sauerwein ${ }^{11}$, Chris Drakeley ${ }^{12}$, Thomas A. Smith ${ }^{2,3}$, Teun Bousema ${ }^{11,12} \&$ Azra C. Ghani ${ }^{1}$
}

Mass-screen-and-treat and targeted mass-drug-administration strategies are being considered as a means to interrupt transmission of Plasmodium falciparum malaria. However, the effectiveness of such strategies will depend on the extent to which current and future diagnostics are able to detect those individuals who are infectious to mosquitoes. We estimate the relationship between parasite density and onward infectivity using sensitive quantitative parasite diagnostics and mosquito feeding assays from Burkina Faso. We find that a diagnostic with a lower detection limit of 200 parasites per microlitre would detect $55 \%$ of the infectious reservoir (the combined infectivity to mosquitoes of the whole population weighted by how often each individual is bitten) whereas a test with a limit of 20 parasites per microlitre would detect $83 \%$ and 2 parasites per microlitre would detect $95 \%$ of the infectious reservoir. Using mathematical models, we show that increasing the diagnostic sensitivity from 200 parasites per microlitre (equivalent to microscopy or current rapid diagnostic tests) to 2 parasites per microlitre would increase the number of regions where transmission could be interrupted with a mass-screen-and-treat programme from an entomological inoculation rate below 1 to one of up to 4 . The higher sensitivity diagnostic could reduce the number of treatment rounds required to interrupt transmission in areas of lower prevalence. We predict that mass-screen-and-treat with a highly sensitive diagnostic is less effective than mass drug administration owing to the prophylactic protection provided to uninfected individuals by the latter approach. In low-transmission settings such as those in Southeast Asia, we find that a diagnostic tool with a sensitivity of 20 parasites per microlitre may be sufficient for targeted mass drug administration because this diagnostic is predicted to identify a similar village population prevalence compared with that currently detected using polymerase chain reaction if treatment levels are high and screening is conducted during the dry season. Along with other factors, such as coverage, choice of drug, timing of the intervention, importation of infections, and seasonality, the sensitivity of the diagnostic can play a part in increasing the chance of interrupting transmission.

Nature 528, S94-S101 (3 December 2015), DOI: 10.1038/nature16040

This article has not been written or reviewed by Nature editors. Nature accepts no responsibility for the accuracy of the information provided.

$\mathrm{P}$ lasmodium falciparum malaria was responsible for an estimated 584,000 (range 367,000-755,000) deaths in 2013, most of which occurred in young children in sub-Saharan Africa'. Although the burden has reduced in response to global efforts to increase the provision of proven malaria interventions such as insecticide-treated bed nets and access to health care and treatment', it remains high. One of the challenges in reducing malaria transmission is the long duration of infection in the human host, which in semi-immune individuals may persist for a year or more ${ }^{2}$. In particular, although infection often leads to disease in naive individuals, those with sufficient acquired immunity can harbour parasites - and hence be onwardly infectious to mosquitoes - without exhibiting symptoms ${ }^{3}$. One option for speeding the decline in transmission could be to target the asymptomatic reservoir of infection ${ }^{4}$ by providing either periodic mass-screen-and-treat (MSAT) programmes, focal MSAT or a reactive strategy in which individuals living in the vicinity of an identified clinical case are screened and treated. However, the extent to which such strategies are able to reduce the infectious reservoir will depend on the extent to which the diagnostic used to identify infected individuals also detects those who are onwardly infectious. Another form of targeting could take place at the population level (for example a village) where mass interventions are deployed if the population prevalence

\footnotetext{
*These authors contributed equally. ${ }^{1}$ MRC Centre for Outbreak Analysis and Modelling, Department of Infectious Disease Epidemiology, Faculty of Medicine, Imperial College London, Norfolk Place, London W2 1PG, UK. ${ }^{2}$ Swiss Tropical and Public Health Institute, Socinstrasse 57, 4002 Basel, Switzerland. ${ }^{3}$ University of Basel, Petersplatz 1, 4001 Basel, Switzerland. ${ }^{4}$ Institute for Disease Modelling, Bellevue, Washington 98005, USA. ${ }^{5}$ Department of Biomedical Sciences, Centre National de Recherche et de Formation sur le Paludisme, 01 B.P. 2208, Ouagadougou, Burkina Faso. ${ }^{6}$ Mahidol-Oxford Tropical Medicine Research Unit, Faculty of Tropical Medicine, Mahidol University, Bangkok 10400, Thailand. ${ }^{7}$ Centre for Tropical Medicine, Nuffield Department of Medicine, University of Oxford, Oxford OX3 7LJ, UK. ${ }^{8}$ National Malaria Center, Ministry of Health, Phnom Penh 12302, Cambodia. ${ }^{9}$ Department of Statistical Sciences, University of Cape Town, Rondebosch 7701, Cape Town, South Africa. ${ }^{10}$ PATH, 2201 Westlake Avenue, Seattle, Washington 98121, USA. ${ }^{11}$ Radboud University Medical Center, 6525 HP Nijmegen, the Netherlands. ${ }^{12}$ London School of Hygiene \& Tropical Medicine, Keppel St, London WC1E 7HT, UK. Correspondence should be addressed to: H. C. S. e-mail hannah.slater@imperial.ac.uk.
} 
a
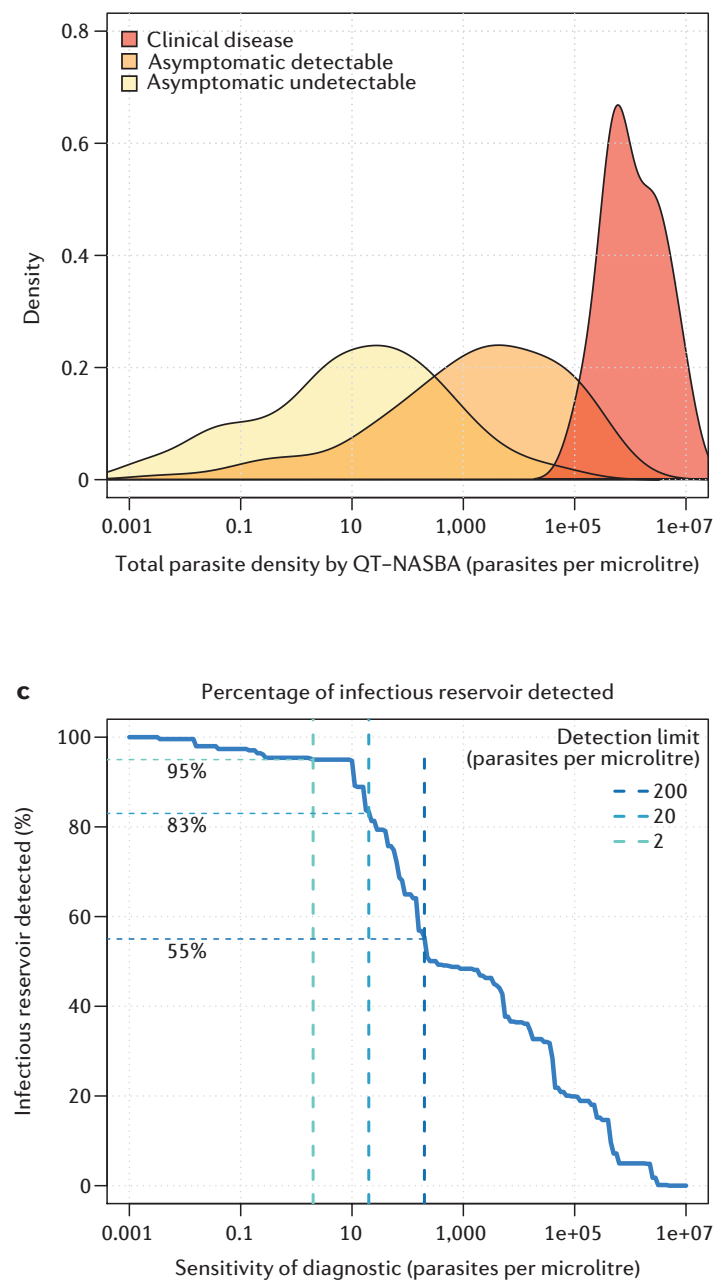

b

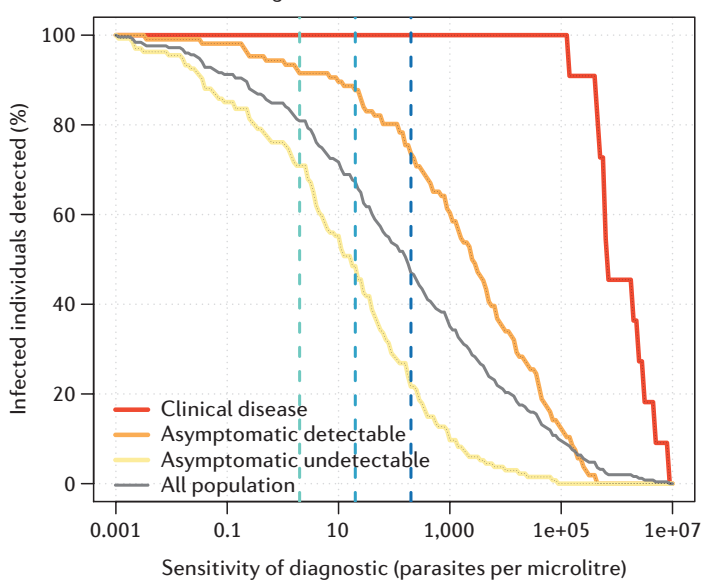

OpenMalaria predictions of percentage

d

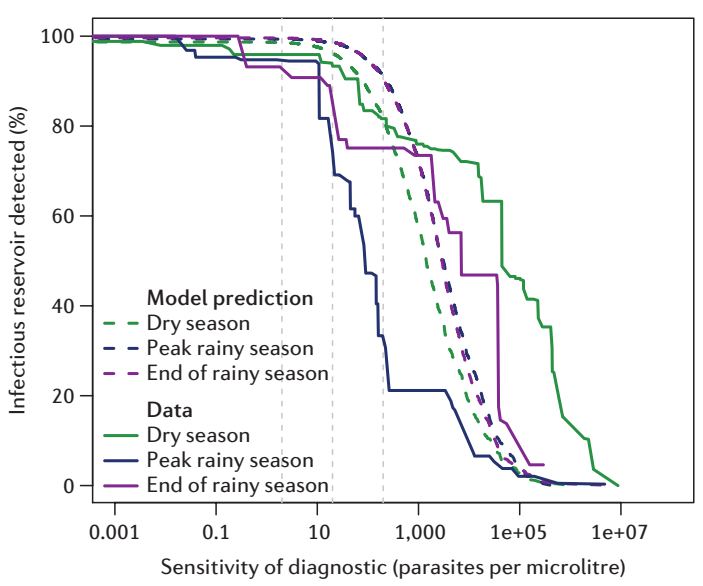

Figure 1 | Percentage of infected individuals and the infectious reservoir detected. a, Parasite density distributions of the three infection groups defined in the statistical analysis section. b, Proportion of infections detected for each infection group for a range of diagnostic thresholds between 0.001 and $10^{7}$ parasites per microlitre. For each value of the $x$-axis we calculate the proportion of each density distribution (from a) that would be detected. $\mathbf{c}$, The proportion of the infectious reservoir of the whole population that would be detected for each diagnostic threshold. This is the combined infectivity to mosquitoes of all individuals with asexual parasite densities above the diagnostic threshold weighted by body size. The dashed vertical lines show the three detection thresholds considered: 200,20 and 2 parasites per microlitre. d, Proportion of the infectious reservoir detected. Comparison of the observed data as shown in $\mathbf{c}$ to a simulation-based approach using OpenMalaria. We simulated the Burkina Faso setting using OpenMalaria to see how well the distributions of parasite density and the contribution to the infectious reservoir could be captured. We assumed that the seasonality followed that reported in Burkina Faso ${ }^{48}$ with 30 infectious bites per person per year in the village of Laye and 300 in the village of Dapélogo, and we assumed that the coverage of treatment for malaria fevers was low. The assumptions about transmission intensity and case management were not crucial to predictions in this range. We weighted each individual's contribution to the infectious reservoir by their body surface area for both the observed data and predictions to account for differential biting rates. The simulated individuals have the same age- and village-distribution as the observed data. The asexual densities were measured using quanitative nucleic acid sequence based amplification (QT-NASBA) whereas OpenMalaria output densities were calibrated to microscopy using the standard method of counting parasite against leukocytes and converting them, assuming 8,000 leukoytes per microlitre ${ }^{49}$. The agreement between microscopy and QT-NASBA densities is not perfect; however, we assume for the purposes of this validation that they are similar because they are two imperfect methods to measure the density of infection.

exceeds a given threshold. In this case, the choice of diagnostic will influence the accuracy of the targeting and the choice of detected prevalence threshold above which the intervention occurs.

Historically, microscopy was the most commonly used diagnostic, both in the clinic to confirm cases, and in the field to estimate the prevalence of asymptomatic infection. Although detection thresholds of 4-20 parasites per microlitre are achievable using a Giemsa-stained thick blood film in a controlled laboratory setting ${ }^{5}$, thresholds of 100-200 parasites per microlitre are more common in field settings ${ }^{6}$. More recently, rapid diagnostic tests (RDTs) have been introduced in the field. These tests detect malaria antigens, most commonly histidine-rich protein 2 (HRP2) and plasmodium lactate dehydrogenase $(\mathrm{pLDH})$, which are antigens produced by most, but not all, $P$. falciparum parasites $^{7-9}$. These diagnostics are easier to use and more reliable than microscopy, but have similar detection thresholds ${ }^{10,11}$. The advent of highly sensitive molecular methods that detect parasite DNA or RNA such as polymerase chain reaction $(P C R)^{12}$ and quantitative nucleic acid sequence based amplification (QT-NASBA) has highlighted that many infected individuals have parasite densities that are too low to be detected either by microscopy or RDTs ${ }^{13,14}$. However, even these methods do not detect all the infections that are present in infected human hosts ${ }^{12,15}$ and they are currently not available as routine field diagnostic tests because they require high-level laboratory facilities for nucleic acid extraction, amplification and detection that are unavailable in many resource-poor endemic settings. Even the most operationally attractive molecular diagnostic available - loop-mediated isothermal amplification 
Table 1 | Gametocyte density, prevalence and proportion of mosquitoes infected for the three infection groups: clinical disease, asymptomatic detectable infection and asymptomatic undetectable infection, and contribution to the infectious reservoir (total infectivity weighted by body surface area).

\begin{tabular}{|l|l|l|l|l|}
$\begin{array}{l}\text { Compartment and percentage of } \\
\text { total infected population }\end{array}$ & $\begin{array}{l}\text { Gametocyte densities } \\
\text { (parasites per microlitre) } \\
\text { of gametocytaemic } \\
\text { individuals (median and } \\
\text { interquartile range) }\end{array}$ & $\begin{array}{l}\text { Proportion of individuals } \\
\text { in compartment that are } \\
\text { gametocytaemic (by QT- } \\
\text { NASBA) }\end{array}$ & $\begin{array}{l}\text { Percentage of } \\
\text { mosquitoes infected } \\
\text { by all the individuals in } \\
\text { compartment }\end{array}$ \\
\hline Clinical disease (4.4\%) & $290.9(181.7,1,169.7)$ & $100 \%$ & $23.1 \%$ & $9.0 \%$ \\
\hline Asymptomatic detectable (42.2\%) & $89.2(15.6,490.1)$ & $87 \%$ & $12.7 \%$ & $62.3 \%$ \\
\hline Asymptomatic undetectable (53.4\%) & $29.8(1.9,128.6)$ & $59 \%$ & $3.9 \%$ & $28.7 \%$ \\
\hline
\end{tabular}

The clinical disease group consists of the $4.4 \%$ of individuals who have the highest parasite densities. Asymptomatic detectable individuals are those that are detectable by QT-NASBA and microscopy, and the asymptomatic undetectable individuals are those detectable by QT-NASBA, but not microscopy.

(LAMP), which involves a simple DNA extraction procedure, isothermal amplification and visual examination of positivity - is currently only used in research settings ${ }^{16}$.

Two recent MSAT trials, which used RDT screening to identify infections, failed to lead to a sustained reduction in malaria parasite prevalence or disease incidence ${ }^{17,18}$. It has been suggested that this could be at least partly due to ongoing transmission from low-density infections that were undetectable by the RDT or by microscopy. For example, in one MSAT study in Zanzibar, just $0.2 \%$ of people tested positive by RDT and received an antimalarial in two rounds 1 month apart, whereas $2.5 \%$ and $3.8 \%$ of people in the two rounds, respectively, were positive by PCR. RDT, therefore, detected just $8 \%$ and $5.3 \%$ of PCR-positive infections in each of the rounds, respectively ${ }^{17}$. A follow-up study in this setting found that LAMP was able to detect 3.4 times more infections than RDT ${ }^{19}$. Village-level targeted mass drug administration (MDA) is being trialled in the Greater Mekong subregion (Cambodia, Laos, Myanmar, Thailand, Vietnam and Yunnan Province in China) with a view to wider implementation ${ }^{20}$. In this setting a random sample of villagers is being screened using a PCR to inform decisions on MDA implementation.

To improve the impact of elimination strategies such as MSAT and targeted MDA, researchers are developing RDTs with improved limits of detection. Trying to establish the appropriate target performance specifications for these tests raises two questions. First, how infectious are individuals with low parasite densities (how much could they contribute to the infectious reservoir)? Second, could the use of a more-sensitive diagnostic increase the ability of an MSAT intervention to achieve local interruption of transmission?

We address these questions from a population perspective using a combination of data analysis and mathematical modelling. Using a data set from a study of the human infectious reservoir for malaria in a high endemic site in Burkina Faso, we estimate the contribution to the infectious reservoir of individuals with different parasite densities and hence the proportion of the infectious reservoir that could be detected across a range of diagnostic sensitivity thresholds. We then use three well-established mathematical models of malaria transmission to assess how these different diagnostic sensitivities could improve the prospect of malaria elimination in an African and an Asian context.

\section{METHODS}

Data. The data set used consists of information on 130 participants from four age groups ( $<5$ years, $5-14$ years, $15-30$ years and $>30$ years) in the villages of Laye and Dapélogo in Burkina Faso ${ }^{21}$. Study participants provided 307 venous blood samples at the start of the wet season $(n=104)$, the peak of the wet season $(n=100)$ and in the subsequent dry season $(n=103)$. Age- and village-matched replacements were sought if individuals were lost or refused follow-up visits. Only individuals with serious acute disease, including severe clinical malaria, were excluded from participation. Finger prick blood samples were used for the preparation of microscopy slides, of which 100 microscopic fields were double read for asexual parasites and gametocytes. Nucleic acids were extracted from $100 \mu \mathrm{l}$ venous blood samples and QT-NASBA was used to detect all parasite stages based on $18 \mathrm{~S}$ ribosomal RNA and specifically to detect mature gametocytes based on Pfs 25 messenger RNA. The sensitivity of QT-NASBA was set to 0.01-0.02 parasites per microlitre for both 18S rRNA and Pfs $25 \mathrm{mRNA}^{22}$. Parasite and gametocyte densities were estimated in relation to a standard asexual and gametocyte stage $\mathrm{V}$ dilution series ${ }^{23}$.
Venous blood samples of $3 \mathrm{ml}$ were fed to locally reared 4- to 5-day-old female Anopheles gambiae sensu stricto mosquitoes ${ }^{24} ; 7$ days after feeding a mean of 39.3 (range 14-65) fully fed mosquitoes were dissected in 1\% mercurochrome and screened for oocysts.

Statistical analysis. To estimate the infectious reservoir, we categorized the sampled individuals into four groups: clinical disease, which was defined as individuals with the highest asexual parasite densities ( $>140,000$ parasites per microlitre); asymptomatic detectable, which was defined as individuals who were positive using QT-NASBA and microscopy; asymptomatic undetectable, which was defined as individuals who were positive by QT-NASBA and negative by microscopy; and uninfected individuals who were negative by QT-NASBA and microscopy. We define positive QT-NASBA as all individuals positive for $18 \mathrm{~S}$ because this indicates the presence of asexual parasite stages. For the purposes of this analysis, we make the assumption that QT-NASBA is the gold standard diagnostic. Of the 1,095 mosquitoes that fed on 28 individuals in the group negative by QT-NASBA and microscopy, only 1 became infected $(<0.1 \%$ of mosquitoes, $3.5 \%$ of humans), indicating that the uninfected individuals have extremely low infectiousness to mosquitoes. For each of the other groups we calculated the mean gametocyte density of gametocytaemic individuals, the proportion that were gametocytaemic using QT-NASBA, the mean gametocyte density conditional on the presence of gametocytes and the proportion of fed mosquitoes infected. The contribution of each group to the infectious reservoir was calculated as the total percentage of mosquitoes infected by each group weighted by the number of people in each group and by the relative body surface area (based on age) of those individuals. Non-parametric (Wilcoxon) tests were used to test statistical significance of differences in parasite densities between the groups. Differences in the proportions of gametocytaemic individuals and mosquitoes infected were assessed using logistic regression models using random effects to allow for repeated sampling of some of the same individuals in the different surveys.

In the models, for a given diagnostic detection limit, the proportion of the infectious reservoir that is detectable is calculated as the combined onward infectivity to mosquitoes of all individuals with parasite densities above the threshold (weighted by body surface area) divided by the infectious reservoir of the total population.

Mathematical models. Three established malaria transmission models were used to evaluate how different diagnostic thresholds would impact on the likely success of MSAT strategies. The first two models (the Imperial College model and OpenMalaria, which was developed by the Swiss Tropical and Public Health Institute and the Liverpool School of Tropical Medicine) were used to explore the impact of MSAT in an African context. The third (mathematical and economic modelling (MAEMOD) developed by the Mahidol Oxford Tropical Medicine Research Unit) was used to explore the impact of targeted MDA in an Asian context. Full mathematical details of each of the models, the process of fitting to data and their validation against further field data are given in refs 4 , 25-33. We summarize how the models capture the infectious reservoir. In all three models, we define infectivity as the probability of an individual infecting a feeding mosquito, and the infectious reservoir is defined as the sum of the infectivity of the whole population multiplied by the probability of being bitten, which is based on the body surface area of the individual. 

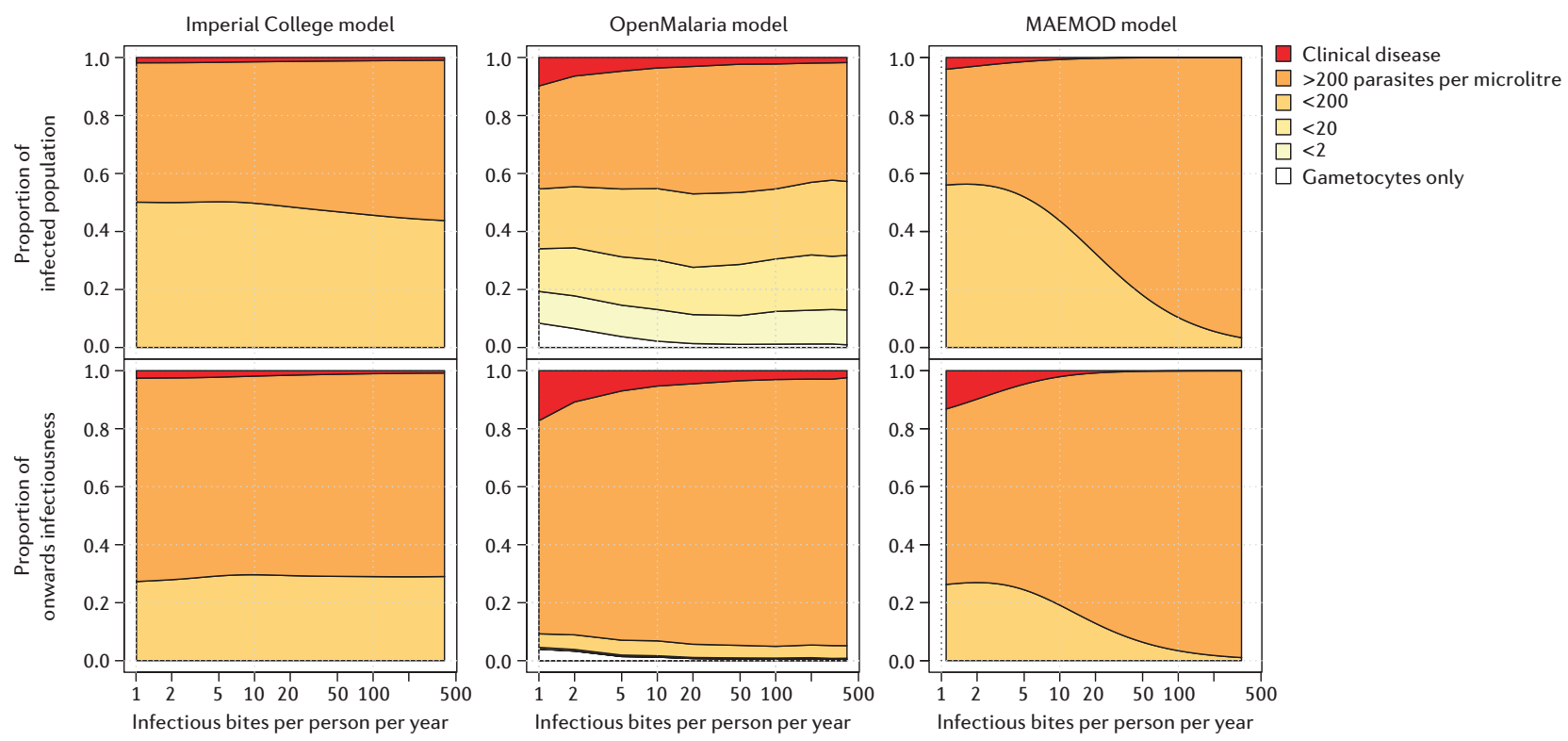

Figure 2 | Model predictions of the proportion of infected individuals in each class and their contribution to the infectious reservoir. Proportion of the infected population that have clinical disease (red), asymptomatic infection detectable by microscopy (orange) and asymptomatic infection not detectable by microscopy (yellow), and their contribution to the infectious reservoir for a range of entomological inoculation rates between 1 and 400 . We predicted the proportion of infected individuals with infections detectable by microscopy for a range of transmission intensities with the three models, assuming the common inputs: stable transmission, constant seasonality, little case management and no other interventions present. The 'gametocytes only' compartment refers to individuals with recently cleared asexual parasites, but persisting gametocytes.

\section{Imperial College}

We used a deterministic age-structured population-level transmission model that tracks three infected compartments of the population - clinical disease, patent asymptomatic infection (identifiable by microscopy), and sub-patent (sub-microscopic) asymptomatic infection ${ }^{29}$. Onward infectivity to mosquitoes is highest in clinical disease, intermediate in patent asymptomatic infection and lowest in sub-patent infection parameterized by fitting to four earlier mosquito feeding studies ${ }^{29}$. As the model does not explicitly track parasite density, we adapted this structure to capture a wider range of diagnostic sensitivities by using results of the data analysis to define the proportion of infections that are detectable at a given parasite threshold within each of the infected compartments. An individual's infectivity is determined by their malaria infection status 10 days previously, to capture lags in gametocyte production.

\section{OpenMalaria}

We used an individual-based stochastic comprehensive model of malaria epidemiology 28,34 . In this model, a population of individuals is updated at 5-day time steps with model components representing new infections, parasite densities, acquired immunity, morbidity, mortality and infectivity to mosquitoes. The course of parasite densities over an infection in a non-immune individual is described using historical data from patients deliberately infected with $P$. falciparum as treatment for neurosyphilis. Immunity to asexual parasites is derived from a combination of cumulative exposure to both inoculations and parasite densities, and maternal immunity ${ }^{26}$, and acts to reduce the densities. The model was fitted to aggregated data from several data sets but not to longitudinal patterns of densities within individuals ${ }^{26}$. Several different variants of this model are available. For this study we use the base model from the overall ensemble ${ }^{34}$. An individual's infectiousness to mosquitoes is related to a weighted sum of their recent parasite densities 10, 15 and 20 days previously, allowing time for gametocytes to develop and circulate ${ }^{27}$. The probability of infecting a feeding mosquito is given by the probability that at least one male and one female gametocyte would be taken up in the blood meal, and the model is fitted to the data of artificial feeding carried out on the patients with neurosyphilis treated using malaria therapy and then scaled using field data to account for the difference between infectivity in experimental and field conditions ${ }^{25,27}$. An individual who has recently been treated or whose infection ended naturally may have no asexual parasites, but continue to be infectious for up to 20 days. The proportion of individuals detected by a diagnostic test, and the corresponding contribution by these individuals to the infectious reservoir, is directly recorded.

\section{MAEMOD}

We used a deterministic model for the transmission of $P$. falciparum malaria similar to those previously described ${ }^{32}$ with four infection classes: severe; clinical; asymptomatic and detectable by microscopy; and asymptomatic and undetectable by microscopy. Each infection class has a distribution of parasitaemia associated with it, which is used to estimate the sensitivity of various diagnostic tests. Each infection class also has an infectiousness associated with it based on infectivity data. We assume that treated individuals test positive for HRP2 after clearance of asexual parasitaemia for different durations, depending on the detection limit of the test.

Simulated scenarios. We considered two implementation scenarios: an MSAT programme in an African setting (Zambia), and targeted MDA in Southeast Asia (Cambodia). The former represents a setting in which malaria transmission is moderate ( $15 \%$ parasite prevalence measured by microscopy in children under 5 years in 2012) ${ }^{35}$, whereas the latter is an area of low prevalence $(<1 \%$ parasite prevalence across all ages measured by microscopy in 2010) ${ }^{36}$. Three diagnostic detection thresholds were considered: 200, 20 and 2 parasites per microlitre. The highest level was chosen to reflect the diagnostic threshold for microscopy and widely used rapid diagnostic tests, although more modern RDTs may have a sensitivity closer to 40 parasites per microlitre. The lower thresholds were chosen to reflect log orders of difference, while remaining a feasible target product profile for a new diagnostic test based on current technologies. A diagnostic threshold of 2 parasites per microlitre is currently achieved by LAMP and nested PCR ${ }^{12}$.

\section{MSAT in an African setting}

The frequency, coverage and artemisinin-based combination therapy used were based on the current operational strategy being implemented in Zambia. A single seasonality profile was used based on average rainfall patterns in Zambia between 2002 and 2009 obtained from the US Climate Prediction Center ${ }^{37}$. We assumed three rounds of MSAT were conducted 1 month apart during the dry season and repeated annually for a maximum of 8 years. 
a PCR prevalence after 8 years of mass treatment

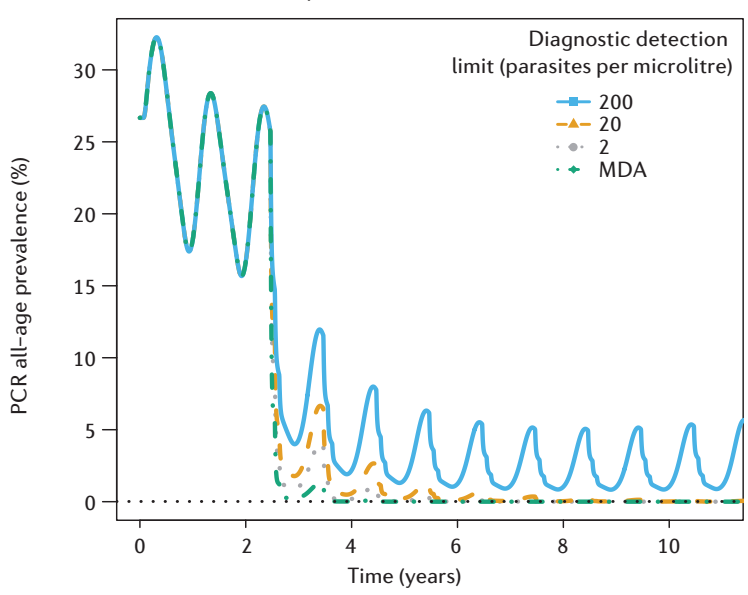

b PCR prevalence (zoom-in) after 8 years of mass treatment

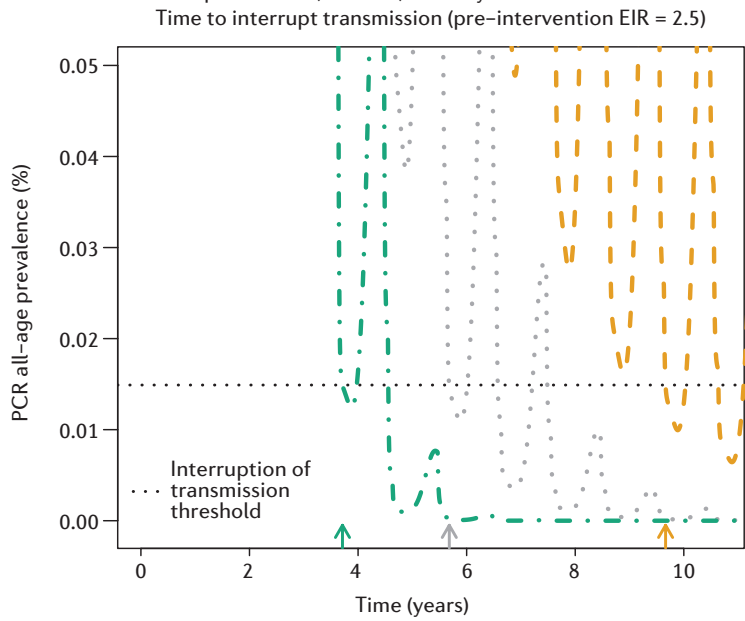

Figure 3 | Example simulation of time to interrupt transmission. a, Time series plots from the Imperial College model showing the impact of 8 years of intervention that consists of three rounds of mass screen and treat (MSAT) or mass drug administration (MDA) a month apart during the dry season with dihydroartemisinin and piperaquine at a coverage of $80 \%$. The entomological inoculation rate is assumed to be 2.5. The blue, yellow and grey lines show the impact of MSAT using a diagnostic with a sensitivity of 200, 20 and 2 parasites per microlitre. The green line shows the impact of MDA implemented with the same timing and coverage as MSAT. The dashed line shows the prevalence threshold at which, if prevalence remains below this line for 50 consecutive days, we claim that transmission is interrupted. $\mathbf{b}$, Enlarged version of a showing the interruption of transmission prevalence threshold. The arrows show the time at which interruption of transmission is achieved and the colours of the arrows correspond to the prevalence curves. EIR, entomological inoculation rates; PCR, polymerase chain reaction.

All treatments were assumed to be with dihydroartemisinin and piperaquine, which has high efficacy in clearing parasites (95\%) and a duration of protection of approximately 30 days $^{38}$. We assumed $80 \%$ coverage of individuals at each round with no correlation between those receiving the drugs between each round, meaning that an individual has a $99 \%$ chance of being treated at least once per year. The simulations were undertaken for a range of assumed baseline transmission levels for Zambia based on entomological inoculation rates (EIRs) between 0.5 and 20 infectious bites per person per year with no imported infections from other regions.

The Imperial College model assumes that the proportion of each infected compartment that is detectable for a given diagnostic threshold is unchanged as prevalence decreases. In this model interruption of transmission is defined a

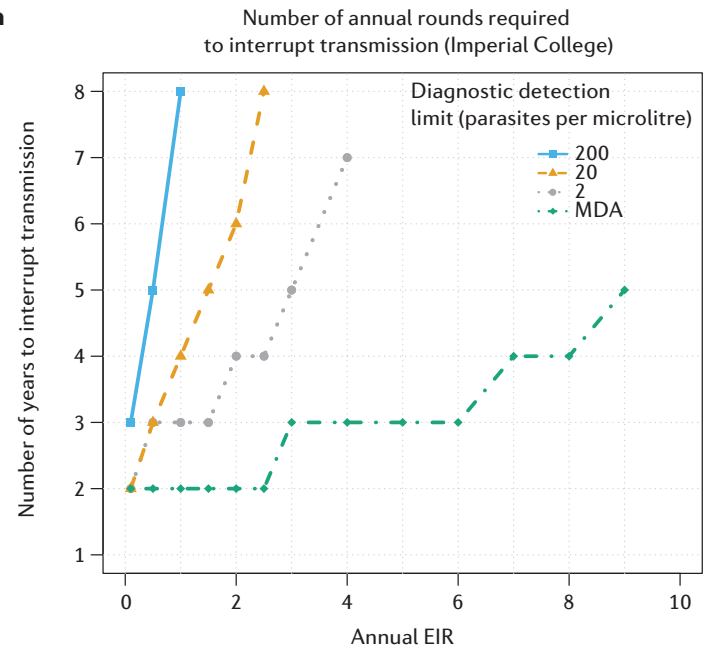

b

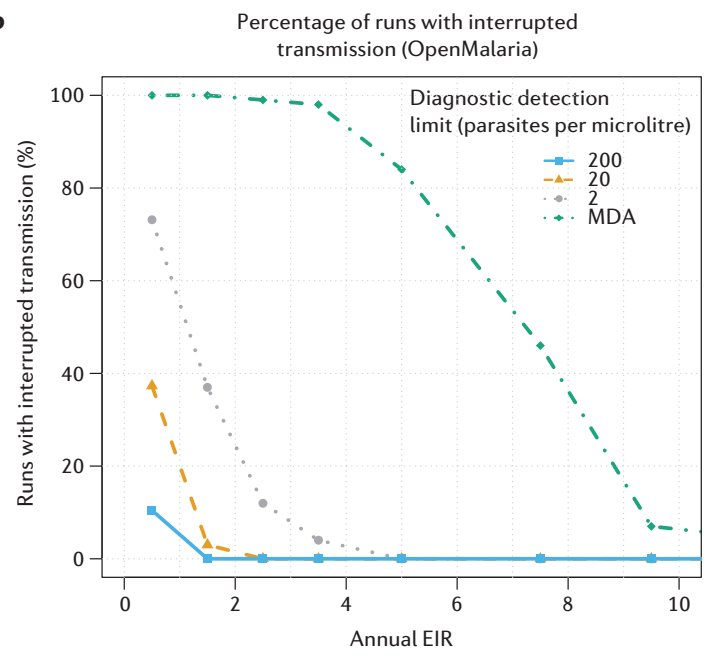

Figure 4 | Predictions of interruption of transmission for mass drug administration (MDA) and mass screen and treat (MSAT) with three diagnostic detection limits. a, Number of annual rounds required to interrupt transmission for a range of entomological inoculation rate values between 0.5 and 9.5 predicted by the Imperial College model. $\mathbf{b}$, The probability of interrupting transmission by transmission intensity and diagnostic sensitivity predicted by OpenMalaria. We simulated scenarios for each effective transmission intensity of 10,000 individuals with no imported infections, no correlation in who receives each dose and low case-management. Because OpenMalaria is stochastic, we ran 100 simulations at each transmission intensity and present the proportion of scenarios in which transmission was interrupted. We chose one point in time to represent the results, after four years of MSAT. $\mathbf{a}$ and $\mathbf{b}$ assume three treatment rounds per year 1 month apart in the dry season.

to have occurred once the PCR-detected prevalence for all age groups has been sustained below $0.0149 \%$ for 50 consecutive days in the absence of importation. This corresponds to a $99 \%$ probability that one or fewer people are infected in a population of 1,000 people ${ }^{39}$. In OpenMalaria, interruption of transmission is achieved when there are no infected individuals in a simulation. We ran 100 OpenMalaria simulations - each with a population size of 10,000 people - for each scenario to predict the probability of interrupting transmission. We did not carry out a formal model comparison.

\section{MDA in Southeast Asia}

At present, targeted MDA is considered the most promising strategy to stop the spread of artemisinin-resistant $P$. falciparum parasites in the Greater Mekong subregion ${ }^{40}$. In the Southeast Asian setting, targeting populations at the village level is being trialled ${ }^{20}$. Villages are screened to measure their 
Predicted prevalence by each diagnostic: with treatment of clinical cases

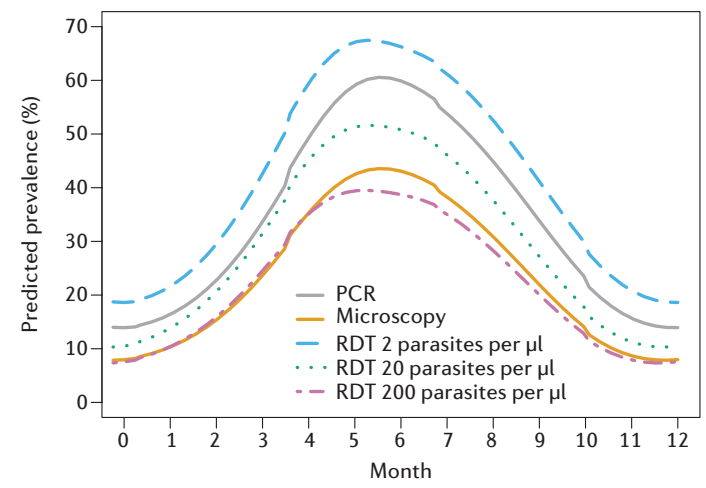

Figure $\mathbf{5}$ | The predicted seasonal pattern of prevalence by polymerase chain reaction (PCR), microscopy and rapid diagnostic test (RDT) (with detection thresholds of 200, 20 and 2 parasites per microlitre) over 1 year in a highprevalence Cambodian setting. The results show the output from the MAEMOD model with high-coverage treatment of clinical cases.

prevalence and then if they are above a given threshold, they are selected for MDA. This is performed in the context of enhanced detection and treatment of clinical cases and high-coverage vector control. Currently, the diagnostic used is PCR. We used the MAEMOD model to investigate how using an RDT with a sensitivity of 200, 20 or 2 parasites per microlitre would affect the identification of villages. Simulations were performed for a single-peaked high-transmission Cambodian setting ${ }^{41}$ where a village (with a population of roughly 1,000 individuals) can have a prevalence as high as $70 \%$. This prevalence would correspond to an EIR of 3.5 per year (assuming little or no treatment of clinical cases) or 4.5 per year (assuming high coverage of treatment of clinical cases) and single-peaked seasonal transmission. The predicted prevalence in a village over time with the different diagnostics was used to assess the ability of the diagnostic to select a village for targeted MDA.

\section{RESULTS}

Table 1 summarizes the infection status and infectivity of the 128 participants who were sampled once, twice or three times over one annual transmission cycle in Burkina Faso. Of the 277 samples, 251 were found to be positive by QT-NASBA. Overall, 4\% (11) of infections were considered to have clinical disease (associated with high parasite density), 42\% (106) were considered to have asymptomatic detectable infections and 53\% (134) were asymptomatic and negative under microscopy, but positive by QT-NASBA (referred to as asymptomatic undetectable). The remaining 26 samples were negative by both QT-NASBA and microscopy.

Those with clinical disease had the highest gametocyte and total parasite densities, whereas those with asymptomatic detectable infection had gametocyte and total parasite densities higher than those with asymptomatic undetectable infection (Table 1, Fig. 1a). The infection compartment has a significant effect on the proportion of individuals that are gametocyte positive ( $P$ $<0.001)$. This trend in parasite density and gametocytaemia is mirrored in the infectivity to mosquitoes: those with clinical disease infect a higher proportion of mosquitoes (23\%) than those with microscopically asymptomatic detectable infection (13\%) and those with asymptomatic undetectable infection $(4 \%)(P<0.001)$. In this setting, individuals with asymptomatic undetectable (sub-microscopic) infections made up 53\% of the population and contributed $29 \%$ of the infectious reservoir of the population.

Under all three diagnostic thresholds considered, all individuals with clinical disease would be detected (Fig. 1b, 1c). At a diagnostic threshold of 200 parasites per microlitre, $74 \%$ of the individuals with asymptomatic detectable infection would be detected. Increasing the sensitivity of the diagnostic to 20 or 2 parasites per microlitre increases this proportion to $88 \%$ and $92 \%$, respectively. Similarly, with a sensitivity of 200 parasites per microlitre, only $22 \%$ of individuals with asymptomatic undetectable infection would be detected. This proportion increases to $49 \%$ and $71 \%$ by increasing the sensitivity of the diagnostic to 20 or 2 parasites per microlitre, respectively. Combining these groups and weighting by relative infectivity to mosquitoes, only 55\% of the infectious reservoir is detected using a threshold of 200 parasites per microlitre. We estimate that a new diagnostic with a sensitivity of 20 or 2 parasites per microlitre would be able to detect $83 \%$ and $95 \%$ of the infectious reservoir, respectively.

Figure 1d shows the proportion of the infectious reservoir detected in the Burkina Faso setting as predicted by the OpenMalaria model (the model based on parasite densities, albeit calibrated to microscopy rather than QT-NASBA densities, and therefore the model from which such predictions can be made). These generally compare well to those observed in the data (Fig. 1c,d), but with less variability with season than observed, and a slightly smaller contribution to the infectious reservoir at low parasite densities. The detectability of infections in the data was generally lowest at the peak of the transmission season, but this pattern of seasonality was not evident in OpenMalaria.

Figure 2 shows the predicted proportion of infected individuals in each infection class for a range of transmission intensities assuming no seasonality in transmission for the three models. In both the Imperial College and OpenMalaria models, these proportions are predicted to remain reasonably constant across a range of transmission intensities, although age-specific predictions show a decrease in the proportion of younger children with sub-patent infections with transmission intensity and an increase in older adults in both models (results not shown). By contrast, the MAEMOD model suggests a higher proportion of patent infection at high-transmission intensities. This difference is probably due to the data sets used for validation, with the MAEMOD model being calibrated for low-transmission settings. In both the Imperial College and MAEMOD models, at low transmission, the proportion of individuals with clinical malaria, patent infection and sub-patent infection, and the infectivity from these groups closely match those observed in the Burkina Faso data (Table 1). The OpenMalaria model suggests a similar proportion of the population in the patent and sub-patent categories (assuming a sensitivity of microscopy close to 200 parasites per microlitre), but predicts that a greater proportion of the infectious reservoir comes from those with patent infection. It also predicts a lower proportion of the infectious reservoir from sub-patent infections under the assumption of no seasonality. However, OpenMalaria fares reasonably well when the scenario inputs are tailored to the high-transmission, highly seasonal setting (Fig. 1d). Two of the models (Imperial College and OpenMalaria) were used to simulate the impact of MSAT strategies in a Zambian setting. Figure 3 shows an example of the time to interruption of transmission with a pre-intervention EIR of 2.5 using the Imperial College model. Here the model predicts MSAT using a diagnostic test with a detection limit of 200 parasites per microlitre would not achieve interruption of transmission, even after 10 years. Increasing the sensitivity of the diagnostic to 20 or 2 parasites per microlitre would achieve interruption of transmission after 10 and 6 years, respectively. The Imperial College model predicts that repeated MSAT using a diagnostic with a detection limit of 200 parasites per microlitre only interrupts transmission in areas with an EIR below 1 (Fig. 4a). Increasing the sensitivity to 20 or 2 parasites per microlitre interrupts transmission in areas with EIRs up to 2.5 and 4, respectively. Increasing the sensitivity of the diagnostic also reduces the number of rounds required to interrupt transmission. A similar pattern is predicted by the OpenMalaria model: interruption of transmission is rare using a diagnostic with a sensitivity of 200 parasites per microlitre at an EIR greater than 1 (Fig. 4b). Increasing the sensitivity to 20 or 2 parasites per microlitre increases the probability of interruption at an EIR of 1 , and at a sensitivity of 2 parasites per microlitre increases the range at which interruption could occur up to an EIR of 4. Thus, in a setting with a low EIR with a seasonality pattern from Zambia, both an increase to 20 or to 2 parasites per microlitre could improve the probability of interruption and expand the range of settings in which this could be feasible. However, it should be noted that in both models MDA is predicted to be substantially more likely to interrupt transmission and potentially to increase the range of transmission intensities at which mass treatment could interrupt transmission.

Figure 5 shows the output from the MAEMOD model applied to a Cambodian setting. Owing to the persistence of HRP2 following treatment, this model reflects the seasonal variability of an RDT in predicting prevalence because when treatment levels are high, there will be a higher proportion of HRP2-positive individuals with cleared infections. This will have an impact on the ability 
of the test to detect villages suitable for MDA on a pre-screening. If there are high levels of detection and treatment of clinical malaria, the model predicts that a diagnostic with a sensitivity of 200,20 or 2 parasites per microlitre will predict a prevalence closer to the prevalence by PCR at the beginning of the season (when prevalence in increasing) than at the end of the season (when prevalence is decreasing). In this setting a threshold of 20 parasites per microlitre is predicted to be as accurate as PCR at predicting population prevalence during the early malaria season.

\section{DISCUSSION}

The success of test-and-treat strategies to clear the infectious reservoir of $P$. falciparum malaria will depend in part on the extent to which current and new diagnostic tools are able to detect individuals who are infectious to mosquitoes. Our results from a high-endemicity setting in Burkina Faso suggest that individuals with sub-microscopic infection contribute $29 \%$ to the infectious reservoir of the population. Given the close correlation between the sensitivity of RDTs and microscopy ${ }^{10}$ it is likely that a similar proportion of the infected population would not be detected by RDTs. Thus, even if every person in the population is screened and treated at each round (which is operationally very unlikely), just under one-third of the infectious reservoir of the population would remain untreated. There is clearly a need to develop more sensitive diagnostic tools if MSAT strategies are to be successfully deployed in the field.

By stratifying the data by parasite density, we were able to relate parasite density thresholds to the proportion of the infectious reservoir captured. At a threshold of 200 parasites per microlitre, only $22 \%$ of individuals with infection that is undetectable by microscopy have parasite densities measured with QT-NASBA above this threshold. Improving the diagnostic sensitivity to 20 or 2 parasites per microlitre increases the proportion of successfully identified infected individuals to $49 \%$ and $71 \%$, respectively. It should be noted that these results are specific to the Burkina Faso study site - an area with very high and very seasonal transmission. Further research is required to understand how the results might be modified at lower transmission, in less seasonal settings or in settings with declining transmission. Similarly, possible associations of age with the detectability and transmissibility of infections, as well as with exposure to mosquitoes, requires further study ${ }^{42}$.

Not all infectious individuals need to be detected and treated to reduce the effective reproduction number to below 1 . At low endemicity, the effective reproduction number (that takes into account the impact of all interventions) will be closer to 1 and hence the proportion of infections that need to be detected and treated will be lower than at higher endemicity. At a given frequency of MSAT rounds, there is therefore a critical threshold of infectivity that needs to be detected to reduce the reproduction number to below 1 .

Using two different transmission models applied to an African setting with seasonal transmission, our results demonstrate that MSAT using a diagnostic threshold of 200 parasites per microlitre could interrupt transmission at an EIR of between 0.5 and 1 if operational coverage is high and the intervention is continued for 8 years. By increasing the sensitivity of the diagnostic tenfold this is increased to an EIR of between 0.5 and 2.5, whereas increasing the sensitivity 100 -fold would expand this to an EIR of between 2 and 4. Large areas of southern and eastern Africa have EIR values below 4, indicating that repeated MSAT programmes with a high-sensitivity diagnostic test of the order of 2 parasites per microlitre could be considered as an intervention in these areas ${ }^{43}$. However, the EIR in Burkina Faso is much higher than 4, suggesting that MSAT would not be effective even with a highly sensitive diagnostic test (as confirmed in a recent trial ${ }^{17,18}$ ). Equally, using a higher sensitivity diagnostic could reduce the number of treatment rounds required to interrupt transmission, thus reducing the operational and financial requirements and potentially increasing the community acceptance of the intervention. These results do have a number of caveats; in particular, as shown elsewhere, there are a number of factors (including overall coverage levels, the specific drug used, the correlation of coverage between rounds ${ }^{31}$ the degree of importation from other areas ${ }^{44}$, the size of the programme, and the seasonality, timing and coverage of other interventions) that can have a large impact on the success of MSAT and MDA programmes. Similar impacts of diagnostic sensitivity on the probability of elimination have been found ${ }^{45}$.
The costs and operational challenges involved in carrying out the testing may prove more important than diagnostic sensitivity in determining how many infections can be detected and treated ${ }^{46}$. MDA may be a better option if it is acceptable to the local population because the additional prophylactic effect that is obtained by giving the drug to the whole population means that it is always theoretically more effective than MSAT. However, conducting multiple MDA rounds may be unpopular, especially if prevalence and incidence are low. We estimate that in the Zambia-like transmission setting described, MSAT using a diagnostic with a detection limit of 2 parasites per microlitre at a coverage of $80 \%$ is equally as effective as MDA at a coverage of $65 \%$ (results not shown). In addition, the wide-scale use of MDA has been anecdotally associated with parasite resistance ${ }^{47}$, and there are worries that it may also accelerate the spread of artemisinin- and piperaquine-resistant malaria parasites. MSAT may therefore become the preferred option if transmission cannot be interrupted with vector control alone and if diagnostics become sufficiently sensitive to detect the minimum fraction of infectious individuals to impact transmission.

Targeted MDA is being considered in the Greater Mekong subregion. The ability to accurately target villages with high numbers of infected individuals is crucial. We have used the MAEMOD model to compare the prevalence predicted by PCR, microscopy and RDT of three different detection limits. The model predicts that a 20 parasites per microlitre detection limit is as accurate as PCR at predicting population prevalence during the early malaria season and is linked to the presence of HRP2 after successful treatment (which is detectable for longer periods of time for lower detection limits). This leads to recently treated individuals testing positive by RDT after they have cleared their asexual parasites. This could be considered an advantage for measuring population prevalence for the purposes of triggering for MDA because this type of low specificity is an indication of recent infection and is therefore linked to the current true prevalence. This aspect can therefore be exploited for triggered MDA interventions, even though it would be a disadvantage for an MSAT intervention or for clinical malaria diagnosis that leads to the treatment of uninfected patients. Focal MDA could be more acceptable than MDA to local communities because pre-testing confirms that the parasite is present in the community. It could also be more attractive to policymakers, especially in Southeast Asia, as it would mean fewer courses of antimalarial drugs are given to uninfected individuals and more heavily infected communities can be identified and targeted.

QT-NASBA is the most sensitive diagnostic used in the analysis, however, it is unable to detect all infections or infectiousness, illustrated by the fact that 1 out of 1,095 mosquitoes fed on blood from 28 QT-NASBA negative individuals became infected. This may be due to degradation of nucleic acids that disproportionally affect RNA-based diagnostics ${ }^{3}$ or to a limitation in sensitivity regardless of sample handling. Gametocytaemic individuals with sub-PCR infection were also found in a cross-sectional survey in Tanzania using a new ultra-sensitive PCR technique ${ }^{12}$. These findings illustrate that the available diagnostics do not detect all infections that are present in populations and that onward malaria transmission is possible (although with a low probability) from apparently parasite-negative individuals. Importantly, these sensitive molecular techniques are currently unfeasible for large-scale malaria interventions. Diagnostics in development need to strike a balance between being sensitive enough to detect a sufficient proportion of the asymptomatic reservoir, and remaining cheap, useable and having a quick turnaround of results.

1. World Health Organisation. World Malaria Report (WHO, 2014).

2. Bretscher, M. T. et al. The distribution of Plasmodium falciparum infection durations. Epidemics 3, 109-118 (2011).

3. Bousema, T., Okell, L., Felger, I. \& Drakeley, C. Asymptomatic malaria infections: detectability, transmissibility and public health relevance. Nature Rev. Microbiol 12, 833-840 (2014).

4. Águas, R., White, L. J., Snow, R. W. \& Gomes, M. G. M. Prospects for malaria eradication in sub-Saharan Africa. PLoS ONE 3, e1767 (2008).

5. Wongsrichanalai, C., Barcus, M. J., Muth, S., Sutamihardja, A. \& Wernsdorfer, W. H. A review of malaria diagnostic tools: microscopy and rapid diagnostic rest (RDT). Am. J. Trop. Med. Hyg. 77, 119-127 (2007).

6. Bell, D., Wongsrichanalai, C. \& Barnwell, J. W. Ensuring quality and access for malaria diagnosis: how can it be achieved? Nature Rev. Micro. 4, 682-695 (2006).

7. Baker, J. et al. Global sequence variation in the histidine-rich proteins 2 and 3 of Plasmodium falciparum: implications for the performance of malaria rapid diagnostic tests. Malar. J. 9, 1-12 (2010). 
8. Baker, J. et al. Genetic diversity of Plasmodium falciparum histidine-rich protein 2 (PfHRP2) and its effect on the performance of PfHRP2-based rapid diagnostic tests. J. Inf. Dis. 192, 870-877 (2005).

9. Cooke, A. H. et al. Comparison of a parasite lactate dehydrogenase-based immunochromatographic antigen detection assay (OptiMAL) with microscopy for the detection of malaria parasites in human blood samples. Am. J. Trop. Med. Hyg. 60, 173-176 (1999).

10. Banoo, S. et al. Evaluation of diagnostic tests for infectious diseases: general principles. Nature Rev. Micro. S16-S28 (2008).

11. Wu, L. et al. Detecting P.falciparum infections to reduce malaria transmission - how do current diagnostics compare? Nature 528, S86-S93 (2015).

12. Hofmann, N. et al. Ultra-sensitive detection of Plasmodium falciparum by amplification of multi-copy subtelomeric targets. PLoS Med. 12, e1001788 (2015).

13. Okell, L. C. et al. Factors determining the occurrence of submicroscopic malaria infections and their relevance for control. Nature Commun. 3, 1237 (2012).

14. Okell, L. C., Ghani, A. C., Lyons, E. \& Drakeley, C. J. Submicroscopic infection in Plasmodium falciparum-endemic populations: a systematic review and meta-analysis. J. Inf. Dis. 200, 1509-1517 (2009).

15. Bretscher, M. T. et al. Measurement of Plasmodium falciparum transmission intensity using serological cohort data from Indonesian schoolchildren. Malar. J. 12, 21 (2013).

16. Hopkins, H. et al. Highly sensitive detection of malaria parasitemia in a malaria-endemic setting: performance of a new loop-mediated isothermal amplification kit in a remote clinic in Uganda. J. Inf. Dis. 208, 645-652 (2013).

17. Cook, J. et al. Mass screening and treatment on the basis of results of a Plasmodium falciparum-specific rapid diagnostic test did not reduce malaria incidence in Zanzibar. J. Inf. Dis. 211, 1476-1483 (2014).

18. Tiono, A. et al. A controlled, parallel, cluster-randomized trial of community-wide screening and treatment of asymptomatic carriers of Plasmodium falciparum in Burkina Faso. Malar. J. 12, 79 (2013).

19. Morris, U. et al. Field deployment of loop-mediated isothermal amplification for centralized mass-screening of asymptomatic malaria in Zanzibar: a pre-elimination setting. Malar. J. 14, 205 (2015).

20. MORU Tropical Health Network. First Patients Enrolled in Cambodia Targeted Malaria Elimination http://www.tropmedres.ac/first-patients-enrolled-in-cambodia-targeted-malaria-elimination (MORU, 2015).

21. Ouédraogo, A. L. et al. Dynamics of the human infectious reservoir for malaria determined by mosquito feeding assays and ultra-sensitive malaria diagnosis in Burkina Faso. J. Inf. Dis. http://dx.doi.org/ 10.1093/infdis/jiv370 (2015).

22. Schneider, P. et al. Quantification of Plasmodium falciparum gametocytes in differential stages of development by quantitative nucleic acid sequence-based amplification. Mol. Biochem. Parasitol. 137, 35-41 (2004).

23. Walker, M. et al. Improving statistical inference on pathogen densities estimated by quantitative molecular methods: malaria gametocytaemia as a case study. BMC Bioinformatics 16, 5 (2015).

24. Ouédraogo, A. L. et al. A protocol for membrane feeding assays to determine the infectiousness of Plasmodium falciparum naturally infected individuals to Anopheles gambiae. Malariaworld J. 4 (2013).

25. Killeen, G.F., Ross, A. \& Smith, T. Infectiousness of malaria-endemic human populations to vectors. Am. J. Trop. Med. Hyg. 75, 38-45 (2006).

26. Maire, N. et al. A model for natural immunity to asexual blood stages of Plasmodium falciparum malaria in endemic areas. Am. J. Trop. Med. Hyg. 75, 19-31 (2006).

27. Ross, A., Killeen, G. \& Smith, T. Relationships between host infectivity to mosquitoes and asexual parasite density in Plasmodium falciparum. Am. J. Trop. Med. Hyg. 75 , 32-37 (2006).

28. Smith, T. A. et al. Mathematical modeling of the impact of malaria vaccines on the clinical epidemiology and natural history of Plasmodium falciparum malaria: Overview. Am. J. Trop. Med. Hyg. 75, 1-10 (2006).

29. Griffin, J. T., Ferguson, N. M. \& Ghani, A. C. Estimates of the changing age-burden of Plasmodium falciparum malaria disease in sub-Saharan Africa. Nature Commun. 5, 3136 (2014).

30. Griffin, J.T. et al. Reducing Plasmodium falciparum malaria transmission in Africa: a model-based evaluation of intervention strategies. PLoS Med. 7, e1000324 (2010).

31. Okell, L. C. et al. The potential contribution of mass treatment to the control of Plasmodium falciparum malaria. PLoS ONE 6, e20179 (2011).

32. Maude, R. et al. The last man standing is the most resistant: eliminating artemisinin-resistant malaria in Cambodia. Malar. J. 8, 31 (2009).
33. Maude, R. J. et al. Optimising strategies for Plasmodium falciparum malaria elimination in Cambodia: primaquine, mass drug administration and artemisinin resistance. PLoS ONE 7, e37166 (2012)

34. Smith, T. et al. Towards a comprehensive simulation model of malaria epidemiology and control. Parasitol. 135, 1507-1516 (2008).

35. Central Statistical Office \& Ministry of Health. Zambia National Malaria Indicator Survey http://www.nmcc.org.zm/files/FullReportZambiaMIS2012_July2013_withsigs2. pdf (Government of the Republic of Zambia, 2012).

36. National Centre for Parasitology, E.a.M.C. \& Malaria Consortium. Cambodia Malaria Survey, 2010 http://www.malariasurveys.org/documents/CMS\%202010\%20GF\%20 Report\%20(FINAL).pdf (Phnom Penh, Cambodia, 2010).

37. National Weather Service Climate Prediction Center. Africa Rainfall Estimates http:// www.cpc.ncep.noaa.gov/products/fews/rfe.shtml (NOAA, 2006).

38. Okell, L. C. et al. Contrasting benefits of different artemisinin combination therapies as first-line malaria treatments using model-based cost-effectiveness analysis. Nature Commun. 5, 5606 (2014).

39. Slater, H. C., Walker, P. G. T., Bousema, T., Okell, L. C. \& Ghani, A. C. The potential impact of adding ivermectin to a mass treatment intervention to reduce malaria transmission: a modelling study. J. Inf. Dis 210, 1972-1980 (2014).

40. von Seidlein, L. \& Dondorp, A. Fighting fire with fire: mass antimalarial drug administrations in an era of antimalarial resistance. Expert Rev. Anti Infect. Ther. 13, 715-730 (2015).

41. Song, J. et al. Rapid and effective malaria control in Cambodia through mass administration of artemisinin-piperaquine. Malar. J. 9, 57 (2010).

42. Stone, W., Gonçalves, B.P., Bousema, T. \& Drakeley, C. Assessing the infectious reservoir of falciparum malaria: past and future. Trends Parasitol. 31, 287-296 (2015).

43. Gething, P. et al. A new world malaria map: Plasmodium falciparum endemicity in 2010. Malar. J. 10, 1-16 (2011)

44. Churcher, T. S. et al. Measuring the path toward malaria elimination. Science $\mathbf{3 4 4}$, 1230-1232 (2014).

45. Gerardin, J., Ouedraogo, A., McCarthy, K., Eckhoff, P. \& Wenger, E. Characterization of the infectious reservoir of malaria with an agent-based model calibrated to age-stratified parasite densities and infectiousness. Malar. J. 14, 231 (2015).

46. Silumbe, K. et al. Costs and cost-effectiveness of a large-scale mass testing and treatment intervention for malaria in Southern Province, Zambia. Malar. J. 14, 211 (2015).

47. von Seidlein, L. \& Greenwood, B. M. Mass administrations of antimalarial drugs. Trends Parasitol. 19, 452-460 (2003).

48. Ouédraogo, A. L. et al. Seasonal patterns of Plasmodium falciparum gametocyte prevalence and density in a rural population of Burkina Faso. Acta Trop. 105, 28-34 (2008).

49. Shute, G. in Malaria, Principles and Practice of Malariology (eds Wernsdorfer, W. \& McGregor, I.) 781-784 (Churchill Livingstone, 1988).

\section{ACKNOWLEDGEMENTS}

The work of L.J.W. is supported by the Bill and Melinda Gates Foundation (OPP1110500) and the Wellcome-Trust Major Overseas Programme in SE Asia (106698/Z/14/Z). The work of A.R. and T.S. (Swiss TPH) is supported by Bill \& Melinda Gates Foundation grant OPP1032350 and additionally the Gottfried und Julia Bangerter-Rhyner Stiftung and the Novartis Foundation for Medical Biological Research (13A13). P.B. and R.B. (PATH) gratefully acknowledges the support of the Bill \& Melinda Gates Foundation (DIAMETER, OPP1053616). A.L.O. is supported by the Netherlands Organization for Higher Education in the Tropics (grant CF29132006), the Global Good Fund, Bellevue, Washington, USA and the Ministry of Health of Burkina Faso. The work of C.J.D. and T.B. is supported by the Bill \& Melinda Gates Foundation (AFIRM, OPP1034789); T.B. is further supported by a fellowship of the European Research Council (ERC-2014-StG 639776). A.C.G., H.S. and P.W. acknowledge support from the Bill and Melinda Gates Foundation, the Medicines for Malaria Venture, the UK MRC and the UK Department for International Development.

\section{COMPETING FINANCIAL INTERESTS}

The authors declare no competing financial interests. Financial support for this publication has been provided by the Bill \& Melinda Gates Foundation.

\section{ADDITIONAL INFORMATION}

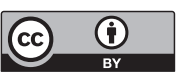

This work is licensed under the Creative Commons Attribution 4.0 International License. The images or other third party material in this article are included in the article's Creative Commons license, unless indicated otherwise in the credit line; if the material is not included under the Creative Commons license, users will need to obtain permission from the license holder to reproduce the material. To view a copy of this license, visit http://creativecommons.org/licenses/by/4.0 